\title{
2019 Undergraduate Big Data Challenge: Big Data of Recreational Drugs
}

\author{
Sponsored by: Roche Canada, the Schulich Foundation, and the University of Calgary HunterHub
}

by

Aazad Abbas, Aleksandra Udovica, Alice Feng, Alice Wu, Alun Stokes, Amenda Arulandoo, Aranyah Shanker, Armaan Mohan, Benjamin Davidson, Benjamin Perks, Bomin Kim, Bowen Ma, Brent Farand, Clement Chan, David Cheng, Emily Leung, Evan Roubekas, Geedhanjali Vivekanandan, Hansi Xu, Harry Wilton-Clark, Herdiljot Sandhu, Ivy Liang, Jaehyun Hwang, Jakob Mawdsley, Jameson Jimmy Dundas, Jennifer Lee, Jennifer Trinh, Jiawei Xu, Jiin Kim, Johan Fernandes, Jonathan Zaslavsky, June Wu, Kashyap Patel, Kathy Khong, Keying Chen, Khaled Gaber, Kimberly Hinz, Lama Abuloghod, Lang Liu, Laura Tang, Liam Connors, Michael K. Lee, Minh Nguyen, Mohammed Albaghdadi, Nicole Ng, Nicole Ying Xi Lim, Nicolle Hua, Nikhil Nair Hariharan, Olivia Li, Pardeep Gill, Patricia Malinksi, Rabjot Aujla, Raza Haider, Reeta Nan, Renna Lee, Richard Mills, Riley Lankshear, Ryan Sandford, Sahanaa Kugathasan, Sahar Lakhani, Said Aoude, Salma Geissah, Shaelene Standing, Shayan Khalili, Shelley Gibbons, Stefano Mezzini, Suzie (Sukyoung) Lee, Taha Elghamudi, Talha Syde, Tali Glazer, Tony Xu, Ty Werbicki, Tyrell Buenaventura, Wenda Zhao, William Hum, Yingshi Wang, Zara Ahmad, Zena Al-Janaby, Zi Chen Jiang

The STEM Fellowship Big Data Challenge, a unique pedagogical experiment, aims to develop computational thinking through an inquiry-based learning experience for undergraduate students. Students engage in data mining, finding hidden patterns and trends in complex, scientific, Big Data using top-notch analytical tools. This year's national challenge provided a multidisciplinary opportunity to investigate the xenobiotic effects of recreational drugs on human health and society through the prism of computational methods while taking into consideration the pharmacometrics and pharmacodynamics of recreational drugs. We hope that the investigation carried out and solutions proposed by these students are taken into consideration in future policy proposals, national and global initiatives.

Teams utilised a diverse array of tools, including but not limited to: R, Python, SAS, SPSS, and Tableau. For all the variation between project themes, it remains that all submissions are of incredibly high quality. Every paper is demonstrative of immense creativity and high potential on the respective team's part.

On behalf of STEM Fellowship, I would like to extend my heartfelt congratulations to all students who participated in the challenge, and I wish them all the best for their future endeavours in research and data science. It has been a privilege for us to witness the analytical capabilities of the next generation of students firsthand, and I am certain all entrants will continue to demonstrate excellence in their respective research careers.

\section{Jaweria Qaiser}

Director, Data Science Education

\section{Dr. Sacha Noukhovitch}

Founder, Executive Director/ Editor-in-Chief

\section{Disclaimer}

These abstracts are provided for all student teams that have submitted project reports by May 23, 2019. The STEM Fellowship Journal editorial board has made every effort to ensure proof and English editing of these abstracts in a limited amount of time, and neither organization as a whole or any of its volunteer members can be held accountable for inaccuracies that may have occurred in the abstract publication. Abstracts are published in alphabetical order per school names of the participating teams. 


\section{Correlation Between the Legalization of Recreational Cannabis and Opioid Overdose Deaths in Colorado and Washington}

Brent Farand, and Richard Mills

MacEwan University

This paper aims to determine if the legalization of recreational cannabis in Colorado and Washington has had an impact on the trends of opioid overdose deaths in these states. Datasets were collected from organizations including the National Survey on Drug Use and Health (NSDUH) and the Centers for Disease Control and Prevention (CDC). The central target of analysis from these datasets is the number of opioid overdose deaths prior to and after the year of recreational cannabis legalization. This analysis is performed using linear and quadratic regression models, comparing the projections of the number of opioid overdose deaths made prior to the year of legalization with the actual number of opioid overdose deaths following legalization. Linear regression models were primarily used with the exception of cases in which a quadratic regression model represented the data more accurately. A confidence interval of $95 \%$ was used for the model projection. Through these methods, the authors found that there was no significant correlation between opioid overdose deaths in Colorado and Washington and the legalization of recreational cannabis. While the actual data of opioid overdose deaths did trend downward in most cases following cannabis legalization, it did not decrease to such an extent that it could not be explained by an error in the model: the data did not fall outside of the confidence interval. The downward trend of the actual data appears to closely follow the previously existing downward trend and varies little from the projections made before the legalization of recreational cannabis. Although the actual data displays a downward trend, the models suggest that the trend is upwards overall. Despite the lack of a strong correlation, it may be too recent to draw a definite conclusion. As more data is collected and more locations legalize cannabis for recreational use, revisiting the topic may yield different conclusions.

Keywords: cannabis legalization, opioid crisis, opioid overdose, opioid trends
Which Factor(s) Among the Unemployment Rate, Provincial GDP, CPI, and Cannabis Consumption Impacts the Opioid Death Rate the Most in Canadian Provinces?

Alice Feng, Bomin Kim, Jennifer Lee, and Reeta Nan McMaster University

The purpose of this paper was to analyze several factors that could have an impact on the death rates from opioid overdose in 10 different Canadian provinces. The Consumer Price Index (CPI), Provincial Gross Domestic Product (GDP), and unemployment rate (\%) were chosen as the macroeconomic factors for analysis because they are directly related to peoples living standards. Cannabis consumption was also analyzed for its influence on the opioid-related death. To evaluate the relationship between each factors and the death rate, R2 values were calculated to analyze the data collected from PubMed, Web of Science, as well as annual government or provincial statistical analysis websites. The analysis of these factors shows that the Canadian provinces with greater $\mathrm{CPI}$ and higher unemployment rate tend to have lower opioid death rate. However, GDP shows a very small positive correlation with opioid abuse. This paper also shows that increasing Cannabis use also reduces the number of people that die due to opioid overdose. However, the correlation between the factors and the death rate is weak overall. Although an overall correlation was observed, a larger data set consisting of data from more than eight years should be used for more accurate research in the future.

\section{A Novel Minimal-Input Feed-Forward Neural Network for Predicting Drug-Drug Interactions Without Knowledge of Drug Structure}

Alun Stokes, Jonathan Zaslavsky, and William Hum McMaster University

The necessity of predictive models in the drug discovery industry cannot be understated. With the sheer volume of potentially useful compounds that are considered for use, it is becoming increasingly computationally difficult to investigate the overlapping interactions between drugs. Understanding this is also important to the layperson who needs to know what they can and cannot mix, especially for those who use recreational drugs - which do not have the same rigorous warnings as prescription 
drugs. Without access to deterministic, experimental results for every drug combination, other methods are necessary to bridge this knowledge gap. Ideally, such a method would require minimal inputs, have high accuracy, and be computationally feasible. We have not come across a model that meets all these criteria [1, 4, 5]. To this end, we propose a minimal-input multi-layer perceptron that predicts the interactions between two drugs. This model has a great advantage of requiring no structural knowledge of the molecules in question, and instead only uses experimentally accessible chemical and physical properties -20 per compound in total. Using a set of known drug-drug interactions, and associated properties of the drugs involved, we trained our model on a dataset of about 650, 000 entries. We report an accuracy of 0.968 on unseen samples of interactions between drugs on which the model was trained, and an accuracy of 0.942 on unseen samples of interactions between unseen drugs. We believe this to be a promising and highly extensible model that has potential for high generalized predictive accuracy with further tuning.

Keywords: drug interaction, neural network, binary classification, minimal-input model, multi-layer perceptron

\section{An Investigation into Potential Predictors for Opioid Abuse in Canada and the U.S.A.}

Herdiljot Sandhu, and Pardeep Gill

McMaster University

Both the U.S. and Canada are in the midst of a public health crisis concerning opioid use. Policy-makers had responded to the crisis by addressing the prescription rates of opioids, yet opioid-related death rates continue to increase. This paper aims to analyze deaths from opioid use based on various variables, including prescription rates and also socioeconomic factors. Namely, mental illness prevalence, obesity, age, GDP per capita, and educational attainment. While there have been studies based on these variables in relation to opioid use, some results have been inconclusive and few have looked for trends across North America. Datasets were obtained from Canadian and U.S. government databases and were analyzed using SAS Studio. A pairwise scatter-plot was constructed to analyze the relationships between the various variables being studied and a model was constructed using multiple linear regression to understand how the various factors interact and to be able to predict opioid overdose deaths. This analysis revealed a positive relation between opioid-related death rates and incidence of major depressive episodes, educational attainment at the Bachelor's level or above, and median age. These results support the need for updated frameworks for treating opioid addicts and preventing death, including addressing their mental health and structural factors associated with use. These results do not necessarily indicate cause but do reveal associations that should be studied further in order identify at-risk populations and work to address the crisis.

Keywords: opioid crisis, socio-economic factors, linear regression

\section{A Multivariate Analysis of Socioeconomic Factors of Opioid-Related Hospitalizations in Canadian Metropolitan} Areas

\section{Kashyap Patel, and Nikhil Nair Hariharan}

\section{McMaster University}

Opioid misuse is a growing public health concern in Canada. Opioid-related morbidity and mortality rates have been increasing. Although various interventions have been proposed, there is been limited national surveillance data about its prevalence in metropolitan areas. As such, understanding potential socioeconomic factors that might affect opioid poisoning hospitalization rates in metropolitan areas is crucial in developing public health policies to curtail the opioid epidemic. The data was extracted from the Canadian Institute for Health Information and the Statistics Canada Census Program. The data was manipulated, analyzed and visualized using $\mathrm{R}$ Studio. Linear and multiple regression analyses were performed. Further statistical tests were performed to evaluate interaction effects between the socioeconomic predictors. The results of the linear and multiple regression analyses were inconclusive due to the low adjusted R2 value. However, a statistically significant correlation was found between marital status and opioid-related hospitalizations in census metropolitan areas. The study found weak but statistically significant correlations between the predictors and hospitalization rates. The results of this study prompt further research into the effect of marital status on opioid misuse. To inform novel public health policies, more conclusive research is required. Some limitations of this study include the lack of data on municipalities in the metropolitan areas and a time-series analysis.

Keywords: opioid, misuse, hospitalization, socioeconomic, metropolitan 


\section{The Solution to the Opioid Overdose Epidemic}

Amenda Arulandoo, and Sahanaa Kugathasan

Trent University, University of Toronto

With various synthetic opioids entering the illicit drug market, Canada has witnessed a steep rise in deaths caused due to overdose in the recent years. Fentanyl, oxycodone and morphine are familiar analgesics in the health care sector that are known for their ability to suppress or alleviate pain symptoms, however, its numerous side-effects and withdrawal symptoms may deem this family of drugs as fatal, when misused or used in high doses. Opioids are used to reach an analgesic state for patients who are experiencing severe pain. Fentanyl has reached a highdemand within healthcare as its used as an effective pain killer. Consequently, the opioid misuse epidemic has also taken several lives as individuals develop an addiction to the drug. There is a need for an alternative drug that reaches an analgesic state, without severe consequences, such as the results of fentanyl: death or respiratory depression. We have researched and compiled an alternate drug derived from fentanyl itself, which has proven to be more effective and less potent, when administered in a reasonable dosage. Instead of binding to the mu-receptors and stimulating dopamine production as fentanyl does, the derivative activates the peripheral receptors to inhibit the feeling of pain. When the alternate drug FF3 is administered using a computationally designed mechanism, it allows the patient to experience the benefits of an analgesic state whilst preventing any lethal side effects. Protonation of the FF3's ligand is imperative to determine its effectiveness in treating pain. To reach analgesia via FF3 administration, the pKa of the ligand must be obtained in order to achieve an acidic $\mathrm{pH}$ value that is close to the injured tissue. As a result of this chemical reaction, the peripheral pain receptors will become activated and block the feeling of pain for the patient. Through computational design, FF3 can successfully act as an analgesic that is much safer to use than fentanyl (with respect to overdosing cases).

Keywords: fentanyl, receptors, addiction, FF3.
Analysis of Substance Risk Perception, Substance Use Initiation, and Substance Use Disorder in Teens

June Wu, and Kathy Khong

University of British Columbia

The initiation of recreational drug consumption is often related to the risk factors that are associated with specific drugs, which can be perceived differently across various age groups. The aim of this study is to investigate the perception of harm associated with recreational drugs, the level of drug use initiation, as well as the actual risk associated with these drugs among teenagers ages 12 to 17 . The four drugs we examined were marijuana, cocaine, heroin, and alcohol. Correlation and regression data analysis, as well as data forecasting was performed using data obtained from the US National Survey on Drug Use and Health for teenage respondents. We found that the perception of risk for recreational drugs from 2002 to 2017 has been fairly constant, with the exception of marijuana. There has been a steady decline in the proportion of teens who perceive marijuana use as highly risky, with our forecast showing that the drug-risk perception for marijuana is expected to decrease to $33.1 \%$ in 2021, which is a 21.8\% decrease from 2002. Overall, higher drug-risk perception is associated with lower initiation of drug use, and higher initiation of drug use is associated with more cases of substance use disorder. Our findings indicate that if the current trends in substance use perception and initiation continues, we may see an increase in marijuana use disorder in the future. As a result, future investigations on the factors that influence youth perception on drug harm, such as education and social pressures, may be required to understand and manage the changes in teen drug use trends.

Keywords: drugs, perception, abuse, big data, analysis

\section{Stress as a Motivation for Recreational Cannabis Use} Armaan Mohan, Benjamin Perks, Shaelene Standing, and Tali Glazer

\section{University of Calgary}

To promote the safe usage of newly legalized cannabis, it is important to understand the factors that influence the decision to consume the recreational drug. Since cannabis was only legalized in Canada in October 2018, many of the long term effects are still unknown. Further studies and analyses are required before any 
conclusions can be made regarding the efficacy of the legalization of cannabis with regards to the intentions outlined in the Canadian Cannabis Act. When examining a dataset of cannabis strains, a treemap demonstrated that strains which induced relaxing effects were associated with higher ratings. This prompted the question of whether stress is correlated to increased cannabis consumption. This problem was investigated using data analysis undertaken in both $\mathrm{R}$ and Python 3 to analyze open datasets and determine possible relationships. From a Canadian mental health data set, Canadian stress was found to primarily stem from work and financial concerns. To connect this to cannabis usage, unemployment and Canadian cannabis consumption data were graphed as a lineplot using the seaborn package for Python 3. The correlation function provided by the pandas package for Python 3 suggested there was a negative correlation between the two; when more people are employed, there is greater consumption of cannabis. This was further supported in an analysis of household spending on cannabis and unemployment rate over time that also found a negative correlation. One explanation is that increased cannabis consumption is a result of greater disposable income from increased employment. In addition, this newfound relationship suggests that stress is an important inducement for cannabis consumption. The interactions between dopamine receptors and the delta-9-tetrahydrocannabinol (THC) in cannabis to induce relaxation are not fully understood, and neither is the effectiveness and safety of using cannabis as a response to stress. Knowing the connection between stress and cannabis consumption may therefore help the government better promote and research safe cannabis consumption.

Keywords: cannabis, stress, Canada, big data, unemployment

\section{An Exploratory Analysis of the Connection Between Opioid Source and Opioid Abuse}

Harry Wilton-Clark, Jameson Jimmy Dundas, Liam Connors, and Ty Werbicki

\section{University of Calgary}

The opioid crisis has become one of the largest public health issues facing the US, with over 33000 annual opioid-related deaths and an associated cost of $\$ 500$ billion. Despite this, significant sources of prescription opioids remain under-characterized, inhibiting the implementation of remedial policies. Using publicly available datasets, we sought to examine the link between sources of opioids and individuals at risk of opioid-related deaths to improve the fundamental population-level understanding of the opioid crisis to better inform policy decisions. Publicly available opioid-related datasets were cleaned, and prominent relationships were ascertained using iterative and parallel machine learning algorithms. We were able to produce a model which identified a significant association between a physician being a frequent gabapentin prescriber, and the likelihood that they are also a frequent opioid prescriber (OR: $28.19-99.9 \% \mathrm{Cl}$ : 13.87-69.02). Previous studies have identified that patients are nearly twice as likely to die of causes pertaining to opioids if they are simultaneously prescribed gabapentin and opioids when compared to opioids alone, highlighting the importance of these findings. As a result, our findings suggest current prescription patterns may not align with the health interests of patients, and urge caution in the coprescription of gabapentin and opioids. Future studies should focus on better elucidating the link between gabapentin and opioids to better inform prescription practices.

Keywords: opioid, gabapentin, Neurontin, prescription, morphine

\section{Opioid Epidemic Patterns in Canada}

Jennifer Trinh, Nicolle Hua, Sahar Lakhani, and Suzie (Sukyoung) Lee

\section{University of Calgary}

The opioid crisis emerged in recent years, resulting in the loss of many lives in North America. Although there have been extensive efforts by the government and communities to protect affected individuals from overdose and death, there have been limited number of published studies that focus on Canadian statistics. Here, we analyzed various of factors within Canada to identify possible relationships with the growing epidemic. The rates of opioid-related morbidity and mortality had an inverse correlation with the size of the population density, as indicated by the greater rates in health districts with smaller population sizes. There have been an increase number of strong opioids available to the public, despite of the decreasing trend of total amount of opioids prescribed in the past years. These findings suggest that the opioid crisis is dependent on public health, opening more doors for further research and action. 


\section{An Epidemiological Approach to Analyzing the Validity of the Substitution Effect in Relation to the Recreational Legalization of Cannabis}

Bowen Ma, Taha Elghamudi, Talha Syde, and Said Aoude University of Guelph

North America is currently in the midst of an opioid epidemic. Illicit use of opiates and other analgesics have resulted in record high numbers of overdose and accidental death. In an effort to curtail the detrimental impact of opiates, public health officials are seeking novel methods to combat this epidemic. The Substitution effect, a theory derived from the field of behaviour economics, proposes a solution whereby increasing access to cannabis and other soft drugs can decrease dependence on hard drugs such as opiates and heroin. In fact, multiple studies in the past have hinted at the positive impacts of legalized medical cannabis programs, i.e. decrease opioid dependence. However, there is still a lack of knowledge within the public sphere regarding the recent legalization of cannabis for recreational purposes. Therefore, the aim of our study is to investigate the various health outcomes surrounding the legalization of cannabis across different states in the U.S. using tools such as multivariate regression in R. The multivariate regression shows that some factors such as death by opiate overdose are not significantly influenced by the consumption of cannabis, where as, Alcohol Use Disorders have demonstrated a significant negative correlation. Although our results are not conducive to supporting the aim of the Substitution effect, they assist in opening up new avenues of exploration.

Keywords: substitution effect, cannabis, drug overdose

\section{Trends in Smoking Incidence and Cigarette Consumption in Canada Within the Last Two Decades}

Kimberly Hinz, and Stefano Mezzini

University of Regina

Tobacco is a sacred plant in many First Nations cultures where it is burned and rarely inhaled in ceremonies and medicinal practices. In the late 15th century, tobacco was brought to Europe where it gradually gained popularity and was made into chewing tobacco and cigarettes. Despite many articles published on their adverse health effects, cigarette smoking is still a fairly common practice today. The purpose of this analysis was to determine if cigarette use is impacted by year, cost of cigarettes, age, age when first smoked, number of people in the household by age category, province, sex, and marital status. Raw datasets of tobacco use surveys for 17 years were obtained through the Ontario Data Documentation, Extraction Service and Infrastructure website (<odesi>). Generalized additive models (GAMs) were used to predict probability of smoking according to several variables. Cost and age had a strong effect on smoking probability and amount smoked. Furthermore, the younger a person began smoking, the more likely they were to smoke later in life and smoke more cigarettes in a day. Whether or not a person smokes and many cigarettes are smoked daily is a complex model.

Keywords: tobacco, smoking, generalized additive models

\section{Current Cannabis Use and Driving Practices Under the Influence of Cannabis in Canadians}

Aazad Abbas, Mohammed Albaghdadi, and Patricia Malinksi University of Toronto

Through the Cannabis Act (2018), non-medical marijuana use was legalized in Canada in October 2018. Cannabis causes acute impairment of psychomotor and cognitive functions that are required for driving, and this impairment may increase the risk of motor vehicle accident involvement. In this time where increasing data is being collected on cannabis use, a current understanding of the cannabis use and behaviour is required. The purpose of this report was to access recent data on cannabis use and impaired driving in Canada and Ontario, and to describe cannabis use and driving behaviours surrounding legalization of the Cannabis Act (2018). Results from the Integrated Criminal Court Survey, National Cannabis Survey, and Crowdsourcing Cannabis Survey were interpreted and described. An increased proportion of Canadians and individuals in Ontario reported to have used cannabis in the past three months. Approximately $39 \%$ of Canadians reported operating a vehicle within two hours of using cannabis. When comparing age groups, individuals aged 25 years and older indicated the highest proportion of cannabisimpaired driving. While protecting youth from inappropriate cannabis access is important, public health efforts should also be sure to focus safe driving campaigns on both adults, in addition to youth.

Keywords: cannabis, driving, national, Ontario 


\section{Comparison of Fatal Recreational Drug Overdoses between Celebrities and Non-Celebrities}

Aleksandra Udovica, Jiin Kim, Renna Lee, and Zara Ahmad University of Toronto

Previous studies have examined drug overdoses among celebrities, but not in comparison to the general population. This study's goal was to analyze whether celebrities have higher fatal overdose rates from recreational drug use than the non-celebrity population. It is often presumed that celebrities engage in more drug-taking to cope with their stressful and taxing lifestyles, which is an important presumption because admired actors and singers, among other entertainers, typically have a social contagion effect with everything they do, as seen in copycat suicides. To test this claim, we gathered a list of American celebrities that fatally overdosed on drugs from 1999 to 2017 (inclusive), as well as the number of overdoses in the general American population during this time frame. We kept certain drugs of interest and excluded less commonly occurring drugs that resulted in overdose, leaving us with opioids, heroin, cocaine, benzodiazepines, psychostimulants, and antidepressants. First, we gathered some descriptive statistics of both populations including gender, and specific professions of celebrities. Then, we used an independent samples t-test to discover if there was a significant difference between fatal overdoses for the celebrity versus non-celebrity population in general and for each drug listed previously from the years 1999 to 2017. A Pearson's correlation analysis was used to find if there was a difference in the yearly trend of overdoses for celebrities versus non-celebrities also during the years 1999 to 2017. Descriptive statistics demonstrated that generally more males fatally overdose for both celebrity and non-celebrity populations, and musicians, athletes, and actors tend to overdose the most in terms of celebrity professions. In addition, our results from the t-test surprisingly showed that non-celebrities fatally overdosed at significantly higher rates than celebrities from 1999 to 2017, as well as overdosed at a significantly higher rate for each individual drug than celebrities during this time frame, except for psychostimulants. The Pearson's correlation analysis also yielded surprising results, with celebrities bearing an insignificant negative correlation between fatal overdoses and years passed between 1999 to 2017, and non-celebrities bearing a significant positive correlation between fatal overdoses and years passed. We concluded that judgmental heuristics may make us believe that more celebrities fatally overdose than non-celebrities, and that this presumption could potentially be problematic because celebrities have a massive influence on society, which could lead the general population to engage in these self- destructive behaviours when coping themselves.

Keywords: recreational drugs, celebrities, non-celebrities, fatal overdose, social contagion

\section{Countries' Control on Cannabis}

Alice Wu, Ivy Liang, and Minh Nguyen

University of Toronto

This paper analyzes the relationship between a geographical location, its prevalence of cannabis use and its corresponding societal factors. Such factors include a country's GDP, health expenditure, life expectancy and democracy index. Despite many people talking about cannabis and its adverse effects in casual conversation, there is not a lot of statistical research on geographical spaces and their respective cannabis use. These correlations are particularly useful in the interest of legalization of marijuana but also general public curiosity. Datasets were obtained from organizations such as the UN Office of Drugs and Crime and The World Factbook - CIA. These reputable datasets were analyzed using software tools from R. Statistical analyses were constructed to interpret the correlation coefficients and general shape of the graph. Such analyses were conducted using large geographical regions as well as individual countries. The datasets indicated a correlation between an individual country's life expectancy, GDP and health expenditure. This analysis could also be used as a guideline for countries who have already legalized cannabis and want to potentially lessen harmful societal effects or countries wishing to legalize cannabis in the future. This could also be applicable to other drugs, whether illegal or recreational such as alcohol. Overall, this study indicates the correlation between a specific geographical location, its prevalence of cannabis use, effects on society and society's effects on cannabis use.

Keywords: cannabis, societal, impacts, GDP 


\section{A Model for Predicting the Severity of Heroin Addiction from Multi-Drug Usage Patterns}

\section{Hansi Xu, Olivia Li, Nicole Ying Xi Lim, and Zi Chen Jiang University of Toronto}

The pharmacological and physiological effects of opioids have been relatively well-researched, however, there has been a lack of focus on the individual user. With the rise of individualized medicine and the current opioid epidemic, there is a need to find an appropriate way to sort opioid abusers based on the severity of their addiction and predict their potential for addiction. We used a Python-based 14 dimensional k-Nearest Neighbors classifier algorithm to analyse multiple survey data sets to determine the severity of heroin abuse of individual users. This model predicts the use of heroin in an individual using their usage history of multiple other drugs and can also be applied to other drug usage data. As the time and survey data response size were limiting factors, future longer studies with more detailed clinical data sets may yield more accurate results for obscure recreational drugs and quantitative variables.

Keywords: addiction, k-Nearest Neighbours, prediction, opioids, multi-drug

Assessment of the Association Between Smoking and Hypertension Status by Machine Learning in United States Jiawei Xu, Keying Chen, Lang Liu, and Yingshi Wang University of Toronto

This report aims to develop a prediction model based on machine learning to analyze the relationship between the hypertension status of a person and his characteristics as well as his smoking habit. It has been previously established that smoking is usually associated with hypertension and we want to visualize such association via our prediction model. The future hypertension status of a person with normal blood pressure can be predicted based on his body factors such as blood test results, gender, BMI and smoking frequencies in our model.

Keywords: hypertension, smoking, machine learning

\section{An Epidemiological Approach to Opioid Overdose for Improved Responsive Efforts}

Khaled Gaber, Lama Abuloghod, Salma Geissah, and Zena AlJanaby

\section{University of Toronto}

Hundreds of individuals are dying on a daily basis from opioidrelated overdoses, with these numbers accelerating from year to year. Opioid abuse is a huge epidemic in North America, which is why healthcare efforts must be made to overcome this tragic crisis. The aim of this study is to assess socioeconomic factors that might contribute to opioid-related overdoses, such as age, gender, homelessness, and veteran status. Additionally, temporal factors are assessed, including seasonal variations and the days of the week. The dataset used for our analysis was obtained from Arizona State University, and consists of opioid-related emergency phone calls with corresponding patient information. A chi squared test was performed to determine whether each of our variables affects the likelihood of an opioid-related overdose. P values were generated for each variable to test for statistical significance, with all functions carried out using Python 3.7. Age, gender and homelessness depicted statistically significant correlations to an opioid overdose, and so did sea-sonal variation. Ages between 25-34, males and homeless people are at a higher risk of being opioid victims. Although this study is based on the city of Tempe, Arizona, conclusions were consistent with literature findings reported by the World Health Organization. Additionally, the methods described can be applied to make the same assessments using different datasets; this study is a proof-of-concept that can be furthered, expanded and applied to other regions. Knowing when, where and who is at most risk allows a better allotting of targeted responses such as Naloxone/ Narcan availability in EMS and awareness campaigns. Hundreds of individuals are dying on a daily basis from opioid-related overdoses, with these numbers accelerating from year to year. Opioid abuse is a huge epidemic in North America, which is why healthcare efforts must be made to overcome this tragic crisis. The aim of this study is to assess socioeconomic factors that might contribute to opioid-related overdoses, such as age, gender, homelessness, and veteran status. Additionally, temporal factors are assessed, including seasonal variations and the days of the week. The dataset used for our analysis was obtained from Arizona State University, and consists of opioid-related emergency phone calls with corresponding 
patient information. A chi squared test was performed to determine whether each of our variables affects the likelihood of an opioid-related overdose. $P$ values were generated for each variable to test for statistical significance, with all functions carried out using Python 3.7. Age, gender and homelessness depicted statistically significant correlations to an opioid overdose, and so did sea-sonal variation. Ages between 25-34, males and homeless people are at a higher risk of being opioid victims. Although this study is based on the city of Tempe, Arizona, conclusions were consistent with literature findings reported by the World Health Organization. Additionally, the methods described can be applied to make the same assessments using different datasets; this study is a proof-of-concept that can be furthered, expanded and applied to other regions. Knowing when, where and who is at most risk allows a better allotting of targeted responses such as Naloxone/ Narcan availability in EMS and awareness campaigns.

Keywords: opioids, overdose, Naloxone, Narcan, chi-squared analysis

\section{Analytical Models Elucidating Specific Interactions of Cocaine Abuse and Socioeconomic Determinants to Health Outcomes}

\section{Laura Tang, Michael K. Lee, Nicole Ng, and Wenda Zhao University of Toronto}

The use of recreational drugs is interlinked with a host of sociological determinants and physiological changes contributing to increased morbidity and mortality. Although the sociological and physical aspects of drug use are both necessary to understand the effects of recreational drugs, many studies focus narrowly on the contribution of a single cause. To examine the causes and effects of recreational drugs at multiple levels within a population, and to potentially identity at-risk populations, we compiled the health, demographic and drug use data of 9971 Americans from the 2015-2016 National Health and Nutrition Examination Survey (NHANES). Linear regression analysis identified known correlates between certain recreational drugs in addition to a novel link between cocaine use and the development of type 2 diabetes mellitus. Based on these findings, we hypothesized that cocaine-mediated metabolic disruptions affect kidney function and ultimately insulin sensitivity. In support of this hypothesis, we identified in our dataset a significant association between urine creatinine, a marker of kidney function and cocaine use.
Additionally, we created a pharmacodynamic model connecting cocaine and other recreational drugs to general health. Our analysis demonstrated combinatorial effects where the abuse of multiple drugs was related to a marked decrease in general health, suggesting a pharmacodynamic interaction. Our findings shine light on the complications of chronic diseases as a drugabuse outcome with the hope of identifying targets to promote responsible use of recreational drugs.

\section{Canadian Opioid Risk Assessment}

David Cheng, Evan Roubekas, Jakob Mawdsley, and Rabjot Aujla

\section{University of Victoria}

The opioid crisis continues to be the most significant public health crisis in Canada. Both federal and provincial governments have taken actions to address the crisis in 2016, however, year over year the deaths and hospitalizations continue to be increasing across the country. Successful intervention frameworks targeted at drug demand reduction have been proven successful in Portugal through mapping of vulnerable geographical areas for prioritising resource allocation. This paper proposes the same approach to reduce drug demand and analyzes available government of Canada data to determine the factors correlated with high opioid risk and to identify the associated areas of vulnerability. The data utilized in this paper included opioid-related hospitalizations and emergency department (ED) visits, mortality's due to opioids and the dispensing trends of prescription opioids. Geographic data was obtained from the 2011 StatsCan census. The dataset was then cleaned, filtered and combined using Tableau Dataprep before using Tableau to obtain visual figures.This study primarily utilized data provided by government organizations such as StatsCan and other government sponsored groups. While the data gathered provides insight into the opioid crisis, more complete and cohesive data would provide increased clarity to opioid crisis. The results of our analysis shows the relationship and rate of increase between multiple factors involved in the opioid crisis. These factors include prescription opioids, gender, age groups and geographical regions.

Keywords: opioid, correlation coefficient, Defined Daily Doses (DDD), Fentanyl 


\section{Implications of Cannabis Prescribed on Opioid Overdose Rates}

Clement Chan, Jaehyun Hwang, Raza Haider, and Tyrell Buenaventura

University of Waterloo

Cannabis is becoming a popular medication that is used to relieve pain. It started primarily as a recreational drug, cannabis has been legalized in various countries to help patients. Canada has become one of the countries that prescribes cannabis along with some parts of the USA. Since cannabis poses less serious side effects than most opioids, it was worth researching whether it will be replacing opioids in the future, and ultimately reduce the number of deaths due to opioid overdose in North America. The number of deaths due to opioid overdose and the number of cannabis patients were analyzed to see if there is a correlation. Through the analysis using graphical SAS software and data organization in Microsoft Excel, it was found that as the number of cannabis patients in many US states increased, the number of deaths due to overdose also increased. As a result. this hypothesizes that medical cannabis will not be fixing opioids crisis in North America in the near future. We can look at some data from States that have legalized cannabis for a longer amount of time, and compare between the rate of cannabis prescribed to patients and the rate of opioid.

Keywords: opioid overdose, cannabis, analgesic drugs

Exploring the Relationship of the Age Canadians First Use Recreational Drugs to the Type of Drugs Used, Demographics, Health, and How Drug Use Affects Their Social Well Being

Benjamin Davidson, Johan Fernandes, and Shelley Gibbons University of Windsor

As you grow older is it possible that you may want to experiment with recreational drugs at a certain point in your life? Could this happen at a specific age? Could we use information about various demographics to identify which age group or which gender or province or even social structure is most susceptible to drug use and its effects? Using the data collected by biennial surveys conducted by Statistics Canada, the relationship of drug use with age, demographics (province, sex, urban/rural area) as well as self reported health and social well being were evaluated to develop an understanding of which group of people are susceptible to drug use and how it affects their well being. While all of the criteria did not show a direct relationship, it was shown that Canadians in this study had a $83 \%$ chance of using one of the drugs in our study at some point in their lives. This number drops to $15 \%$ when only illegal drugs are considered. If a person makes it to twenty years old without using drugs they have $6.8 \%$ chance of using any of the drugs in the study and $4.3 \%$ for illegal drugs. Making it to 25 without using drugs effectively ends their chance of starting to use drugs with a $1.7 \%$ for any of the drugs and $0.09 \%$ for illegal drugs. In addition, the study results indicated that after approximately 17 years old, people who start using recreational drugs are predominantly female. As expected, the use of drugs did show an effect on your well being. The self reported physical and mental health of an individual was higher for those individuals who did not take recreational drugs. In addition, the use of cocaine, hallucinogens, and/or ecstasy affected purchasing a home and a person's financial situation.

\section{Public Health Effects of Cannabis Legalization in Colorado, Washington, Alaska, and Oregon}

Aranyah Shanker, Emily Leung, and Geedhanjali Vivekanandan Western University

With the recent legalization of cannabis in Canada, regulations have been outlined for the distribution of cannabis within each province and territory. Although adverse health effects have been seen with cannabis use, the belief that cannabis is harmless is growing among the public. Therefore, further investigation into the public health and safety concerns of cannabis legalization is imperative. With limited data in Canada regarding the effects of cannabis legalization, our study examined data from the Colorado, Washington, Alaska and Oregon, with information available before and after legalization. When analyzing cannabis-related arrests, no correlation was observed among all 4 states. Calls from major poison control centres in the states of Colorado and Washington showed strong positive correlations with cannabis use. Cannabisdependence displayed a slight positive correlation with increased cannabis use in Washington, while no correlation was seen with Colorado. Although these findings provided some insight into existing trends as a result of legalizing cannabis for recreational use, certain results were inconclusive. Future research to identify factors more strongly influenced by cannabis legalization is 
required to evaluate the effects of cannabis legalization on public health and safety more accurately. In doing so, this information can assist policymakers in Canada to address potential consequences of cannabis legalization more effectively.

Keywords: marijuana, legalization, cannabis, health, safety

\section{Typical Profile for an Accidental Overdose Victim in Connecticut}

Riley Lankshear, and Ryan Sandford

Western University

This study aims to build a character profile for victims of recreational drug overdose, within the state of Connecticut between 2012-2018. Trends within parameters such as age, sex, and race were investigated during the study and cross referenced with data pertaining to Connecticut's demographics to identify any dependencies which may influence the representation of parameters within the overdose population. Additionally, the distribution of drugs among overdose data was examined to determine which drugs were the most likely to be present in future Overdoses. Statistical tools within Python, R, and Tableau were used to explore the data and identify consistent trends and characteristics shared within Connecticut's drug overdose population. The initial analysis concluded that the profile of a drug overdose candidate is a white male, between the ages of 27 and 53. Further analysis of Connecticut's population demographics revealed that the race of overdose victims was distributed in a similar fashion to the race of Connecticut's population, resulting in the conclusion that a candidate's race had little influence on their likelihood of overdose. Other factors such as age and sex were not influenced by population demographics.

Keywords: accidental overdose, recreational drugs, Conneticut overdoses, profile, demographics

\section{A Study into Tacking E-Cigarette Abuse Among Youths}

\section{Shayan Khalili, and Tony Xu}

Western University, University of Toronto

The aim of this paper is to investigate the age and frequency at which youths are introduced to e-cigarettes and to investigate the impact of e-cigarette advertisement on youths. The data was gathered from the US Center for Disease Control and Prevention's National Youth Tobacco Survey and spanned a period of 6 years,
2012 to 2018. Python's Pandas library was used to prepare the data while SAS Viya and Excel were used to visualize the data. This paper concludes students entering high school, aged 14 and 15 , were the most susceptible to trying e-cigarettes and there is a positive correlation between the promotion of electronic cigarettes and first-time e-cigarette smokers. 